\title{
Geographic Variation of Adjuvant Breast Cancer Therapy Initiation in the United States: Lessons From Medicare Part D
}

\author{
John A. Charlson, MDª, Emily L. McGinley, MS, MPH ${ }^{\mathrm{b}}$; Ann B. Nattinger, MD, MPH ${ }^{\mathrm{a}, \mathrm{b}}$; \\ Joan M. Neuner, MD, MPH ${ }^{\mathrm{a}, \mathrm{b}}$; and Liliana E. Pezzin, PhD, JD
}

\begin{abstract}
Background: Drug utilization under Medicare Part D varies significantly by geographic region. This study examined the extent to which geographic variation in Part D plan characteristics contributes to the variation in choice of initial endocrine therapy agent among women with incident breast cancer. Methods: Two-stage multivariate regression analyses were applied to the 16,541 women identified from Medicare claims as having incident breast cancer in 2006-2007. The first stage determined the effect of state of residence on the probability of having an aromatase inhibitor ( $\mathrm{Al}$ ), as opposed to tamoxifen, as initial endocrine therapy. The second stage provided estimates of the impact of state-specific Part D plan characteristics on variation in choice of initial therapy. Results: There was substantial residual geographic variation in the likelihood of using an Al as initial endocrine therapy, despite controlling for socioeconomic status, breast cancer treatment, and other factors. Regression-adjusted probabilities of starting an Al ranged from $57.3 \%$ in Wyoming to $92.6 \%$ in the District of Columbia. Results from the second stage revealed that variation in characteristics of Part D plans across states explained approximately one-third $(30 \%)$ of the state-level variability in endocrine therapy. A higher number of plans with cost-sharing above the mean, greater spread in deductibles, and a greater spread in monthly drug premiums were associated with lower adjusted state probabilities of initiating an Al. In contrast, a higher number of drug plans with monthly premiums above the state mean and higher mean cost-sharing (in dollars) were both positively associated with likelihood of starting on an Al. Conclusions: Study findings suggest that variation in benefit design of Part D plans accounts for an important share of the large and persisting variability in use of Als-the preferred oral therapy for breast cancer.
\end{abstract}

J Natl Compr Canc Netw 2017;15(12):1509-1517 doi: 10.6004/jnccn.2017.7021

The Medicare Prescription Drug, Improvement, and Modernization Act of 2003 authorized optional prescription drug coverage (Part D) for eligible beneficiaries beginning January 1, 2006. Unlike Medicare Parts A and B, Medicare coverage of prescription drugs relies on private plans within the various states; these plans are allowed wide discretion when setting features and prices. In order to ensure some degree of consistency across states, the Part D legislation included substantial regulatory oversight requiring plans to offer actuarially equivalent benefits, provide coverage of certain medi-

From the a Department of Medicine and ${ }^{b}$ Center for Patient Care and Outcomes Research, Medical College of Wisconsin, Milwaukee, Wisconsin. Submitted April 7, 2016; accepted for publication August 2, 2017.

The authors have disclosed that they have no financial interests, arrangements, affiliations, or commercial interests with the manufacturers of any products discussed in this article or their competitors.

Author contributions: Study concept and design: Charlson, Pezzin, cations, and supply coverage information for a single Centers for Medicare \& Medicaid Services (CMS) Web site to facilitate consumer comparison. A number of recent studies, however, suggest that drug utilization under Part D has been uneven, with prescription drug use among Part D enrollees varying significantly by geographic regions. ${ }^{1,2}$

Adjuvant endocrine therapy for breast cancer is a useful model for studying the relationship between pharmaceutical plan characteristics and choice of therapy. For most postmenopausal women with hormone

Nattinger, McGinley, Neuner. Data acquisition: Pezzin, Nattinger. Data analysis and interpretation: Charlson, Pezzin, Nattinger, McGinley, Neuner. Manuscript preparation: Charlson, Pezzin, Nattinger. Critical revision: Pezzin, Neuner, McGinley, Charlson.

Correspondence: John A. Charlson, MD, Center for Patient Care and Outcomes Research, Medical College of Wisconsin, 8701 Watertown Plank Road, Milwaukee, WI 53226. E-mail: jcharlso@mcw.edu 
receptor-positive $(\mathrm{HR}+)$ disease there are essentially 2 options for oral endocrine therapy: tamoxifen, whose efficacy was initially demonstrated in the mid$1980 \mathrm{~s}^{3}$ or an aromatase inhibitor (AI). Beginning in 2005, recommendations from ASCO suggested that adjuvant therapy for postmenopausal women with $\mathrm{HR}+$ breast cancer include an $\mathrm{AI}$, either alone or in sequence after tamoxifen. ${ }^{4}$ For much of the past decade, AI agents have been significantly more expensive than tamoxifen, with average costs exceeding $\$ 300$ USD monthly. ${ }^{5}$ Although AI agents recently became available in generic formulations with a considerably lower cost to the patient, historical patterns of use of breast cancer-specific endocrine therapies provide useful insights into the ways patients respond to various plan features. In addition, given the expanding number of expensive oral chemotherapy agents and the fact that several malignancies, such as chronic myeloid leukemia (CML) and renal cell carcinoma, have multiple oral treatment options available, our study provides an analytical framework that is applicable to other situations.

This article examines the extent to which geographic variation in Medicare Part D plan characteristics contribute to the variation in choice of initial endocrine therapy agent among women with earlystage breast cancer enrolled in Part D. To that end, we first quantified the net contribution of state of residence in women's probability of choosing the more expensive AIs, as opposed to tamoxifen, as the initial hormonal therapy agent. In a second-stage estimation, we examined whether state pharmaceutical plans' characteristics, such as annual AI drug costs, deductibles, premiums, and cost-sharing, significantly explain such state effects.

\section{Methods}

\section{Sample Selection}

The main sources of data for cohort selection were derived from Medicare medical and pharmaceutical claims. Our inclusion criteria consisted of women aged 65 to 89 years old identified from Medicare claims as having undergone incident breast cancer surgery in 2006 or 2007, based on a validated algorithm ${ }^{6}$ applied to nationwide Medicare Parts A and B claims. Subjects were required to be enrolled in Medicare for at least 12 months before the incident breast cancer surgery date in order to enable the measurement of comorbidities. Subjects were further required to have an identified surgeon for their surgery, to have been enrolled in Medicare Part D for 12 months after the date of surgery, and to have started hormonal therapy with an AI (anastrozole, exemestane, letrozole) or tamoxifen during that period (following recommendations from the National Quality Forum and ASCO), leaving a sample of 16,541 women.

\section{Variable Definitions}

Initial (within 12 months from surgery) endocrine therapy drug choice (AI vs tamoxifen) was identified from individual-level pharmacy event records in the Medicare Part D data set. Information on age, race, ? poverty status (defined by enrollment in the Medicaid program or receipt of the federal low-income subsidy [LIS] benefit), and state of residence at the time of incident breast cancer surgery were derived from Medicare enrollment files. The number of comorbid conditions was characterized by examining individual inpatient, outpatient, and hospital provider claims for the 12-month period preceding the breast cancer surgery using the methodology described by Klabunde et al. ${ }^{8}$ Information about initial breast cancer treatment (mastectomy or breast-conserving surgery) and receipt of adjuvant chemotherapy was also obtained from Medicare claims.

Patients' sociodemographic and economic status was further characterized by including a zip codelevel measure of per capita income obtained from the US Census Bureau, ${ }^{9}$ a variable capturing the density of women aged $\geq 65$ years in the county obtained from the Area Health Resource File (AHRF), ${ }^{10}$ and residence in a rural area, defined according to the Core-Based Statistical Area definitions. ${ }^{10,11}$ Countylevel measures of density of radiation oncologists per elderly persons, obtained from the AHRF, ${ }^{10}$ were also considered for inclusion in the analyses as a proxy for access to oncology specialists, a factor potentially associated with differential patterns of care.

To characterize drug plan variation, we assembled information on the Part D Prescription Drug Plan (PDP) available to Medicare beneficiaries in each state and the District of Columbia by manually querying the CMS Web site used by Medicare beneficiaries to find and compare the Part D PDPs available in their areas. ${ }^{12}$ For each plan in each state, information was collected on deductible amounts, 
monthly drug premiums, dollar range of drug cost sharing, and annual drug cost (ie, total out-of-pocket cost to a beneficiary who took only that particular endocrine agent at the recommended dosing for the entire year, excluding plan premiums) for each endocrine agent of interest (anastrozole, exemestane, letrozole, and tamoxifen). ${ }^{5}$ Based on these data, we assessed variability among plans at the state level by calculating 4 measures-mean cost, spread between the top and bottom quartile costs, number of plans with cost below the state mean, and number of plans with cost above the state mean-for each of 5 constructs: (1) monthly drug premium, (2) deductible, (3) upper value of the dollar cost-sharing range, (4) lower value of the dollar cost-sharing range, and (5) cost after deductible for AIs. Finally, we calculated the total number of Part D PDPs and total PDP enrollment in each state. All values were determined for each of the 50 states (except for the District of Columbia).

\section{Statistical Analysis}

Basic descriptive statistics were used to characterize the sample population according to the subjects' sociodemographic and economic profile, health status, and initial endocrine therapy.

Our main analyses relied on multivariate regression models to test the hypothesis of a significant contribution of Part D plan characteristics to residual geographic variation in initial therapy modality. The multivariate analysis proceeded in 2 stages. In the first stage, a multivariate probit regression was used to examine the effect of state of residence on the probability of initiating AI therapy, controlling for the various patient factors described earlier. ${ }^{13}$ In this stage, the goal was to adjust for all available individual and small-level covariates to allow the most conservative estimate possible of residual variation by state for the following stage. Robust standard errors were computed to account for clustering (ie, multiple observations on patients treated by the same surgeon) and heteroskedasticity effects.

The state "fixed effects," captured by each state's estimated coefficient in this first-stage individuallevel estimation, were then used as the dependent variable in the second stage estimation examining the effect of state-level Part D plan characteristics, as well as number of and enrollment in Part D PDPs, on state variation in $\mathrm{AI}$ use as the initial endocrine therapy. Given that we had no a priori hypotheses regarding which specific Part D plan characteristics might explain geographic variation in $\mathrm{AI}$ use, we relied on a forward stepwise selection, and the magnitude/significance of the Wald likelihood ratio test statistic of alternative specifications, to determine the best fit/most explanatory model.

A $P$ value of $<.05$ was considered the critical level to determine statistical significance in the first-stage regression. However, a lower threshold $(P<.15)$ was used in the second-stage fixed-effects regression, wherein the sample size was reduced to 50 states. Associations approaching significance can provide further insight into Part D plan characteristics effects on state variation in therapy initiation drug for which the conventional $P<.05$ level is not realized because of small sample size. All analyses, which were approved by the relevant Institutional Review Boards, were conducted using SAS version 9.3 (SAS Institute Inc., Cary, NC) and Stata version 12.0 (StataCorp LP, College Station, TX).

\section{Results}

\section{Sample Characteristics}

Of the cohort of 16,541 breast cancer survivors who received endocrine therapy in the first year after incident breast cancer surgery, nearly half were aged $<75$ years, most were Caucasian, and $26.8 \%$ had annual incomes sufficiently low to warrant receipt of Medicare D LIS. The cohort was generally healthy, with more than half having no comorbidities. Most received breast-conserving surgery, and $16.7 \%$ were also treated with adjuvant chemotherapy; most used an $\mathrm{AI}$ as their initial endocrine therapy (Table 1).

However, there was substantial variation by state, with the unadjusted state proportion of women initiating hormonal therapy with an AI ranging from $54.1 \%$ in Wyoming to $83.3 \%$ in the District of Columbia (unadjusted results not shown).

\section{Geographic Variation and Other Factors Associated With Initial Al Use}

As shown in Table 1, there were a number of differences between those who initiated an $\mathrm{AI}$ and those who began tamoxifen. Several of the bivariate differences across the 2 groups persisted in the multivariate regression (Table 2). Women who lived in neighborhoods ranking in the top quartile of per capita 
Charlson et al

\section{Table 1. Summary Statistics}

\begin{tabular}{|c|c|c|c|}
\hline & \multirow[b]{2}{*}{$\begin{array}{l}\text { Overall, \% } \\
(n=16,541)\end{array}$} & \multicolumn{2}{|c|}{ Initial Endocrine Therapy Drug } \\
\hline & & $\begin{array}{c}A l,{ }^{\dagger} \% \\
(n=12,654 ; 76.5 \%)\end{array}$ & $\begin{array}{c}\text { Tamoxifen, \% } \\
(n=3,887 ; 23.5 \%)\end{array}$ \\
\hline \multicolumn{4}{|l|}{ Age, $y^{a}$} \\
\hline $65-74$ & 48.9 & 49.2 & 48.2 \\
\hline $75-84$ & 42.2 & 42.3 & 41.9 \\
\hline $85-89$ & 8.8 & 8.5 & 9.8 \\
\hline \multicolumn{4}{|l|}{ Race $^{a}$} \\
\hline African American & 6.4 & 6.5 & 5.9 \\
\hline Hispanic & 3.9 & 4.1 & 3.2 \\
\hline Non-African American, non-Hispanic & 89.7 & 89.4 & 90.9 \\
\hline \multicolumn{4}{|l|}{ Comorbidities $^{\mathrm{a}}$} \\
\hline 0 & 55.8 & 55.2 & 57.8 \\
\hline 1 & 26.8 & 27.1 & 25.9 \\
\hline$\geq 2$ & 17.4 & 17.7 & 16.3 \\
\hline \multicolumn{4}{|l|}{ Type of breast surgery ${ }^{a}$} \\
\hline Mastectomy & 40.5 & 42.4 & 34.3 \\
\hline Breast-conserving surgery & 59.5 & 57.6 & 65.7 \\
\hline \multicolumn{4}{|l|}{ Surgery year } \\
\hline 2006 & 37.8 & 38.0 & 37.2 \\
\hline 2007 & 62.2 & 62.0 & 62.8 \\
\hline Adjuvant chemotherapy ${ }^{a}$ & 16.7 & 19.3 & 8.5 \\
\hline \multicolumn{4}{|l|}{ Poverty status } \\
\hline Low-income subsidy recipient ${ }^{a}$ & 26.8 & 27.9 & 23.2 \\
\hline \multicolumn{4}{|l|}{ Per capita income in zip code ${ }^{a}$} \\
\hline Poorest quartile & 24.1 & 24.0 & 24.2 \\
\hline Middle quartiles & 50.3 & 49.3 & 53.4 \\
\hline Wealthiest quartile & 25.7 & 26.7 & 22.4 \\
\hline Residence in rural county ${ }^{a}$ & 27.8 & 26.5 & 32.1 \\
\hline Density of elderly women in county, mean (SD)a & $0.80(0.23)$ & $0.79(0.22)$ & $0.82(0.23)$ \\
\hline
\end{tabular}

Abbreviation: $\mathrm{Al}$, aromatase inhibitor.

astatistical significance at the $P<.05$ level based on Pearson chi-square test statistics.

income and those who had a LIS were significantly more likely to start on an AI, as were women who received chemotherapy and those who underwent a mastectomy. Women living in rural areas and those residing in a county with a greater density of elderly women were less likely to initiate AI therapy, whereas those residing in a county with a greater density of radiation oncologists were more likely to initiate AI therapy.

Parameter estimates for the first stage estimation were used to compute the adjusted probabilities that a woman living in a given state would start an AI as her initial endocrine therapy, controlling for all other factors. After controlling for clustering and po- tentially confounding variables, such as differences in the subject composition or rurality of states, the adjusted probabilities ranged from a low of $57.3 \%$ in Wyoming to a high of $92.6 \%$ in the District of Columbia (Figure 1). North Carolina was selected as the reference state, because it most closely approximated the unadjusted national mean $(70.6 \%)$ AI use for the sample. Using a $>5 \%$ deviation from the national mean in either direction as threshold, the other states were determined to have AI initiation rates either significantly above (colored in blue), significantly below (colored in red), or not different (within a $5 \%$ margin, colored in grey) from the reference state of North Carolina. These differences represent 
the independent effect of state of residence on choice of initial endocrine therapy, after controlling for initial breast cancer treatment, individual and ecologic measures of socioeconomic status (SES), and the other potential cofounders. A total of 22 states had AI initiation rates significantly higher than the reference state (national mean), whereas 12 states, mostly in the Northwest and Midwest regions, had AI initiation rates significantly below.

\section{State Variation in Initial Endocrine Therapy and Part D Plan Characteristics}

To determine the effect of state-level Part D plan characteristics on state variation in AI use, a second stage estimation was conducted, using the state "fixed effects" from the first stage analysis as the dependent variable. Table 3 shows the results from the best-fitting stepwise linear regression. Variation in characteristics of Part D plans across states explained $30 \%$ of the overall state-level variability in initiating hormonal therapy with an AI versus tamoxifen. States with a higher number of plans with monthly premiums above the state mean had higher rates of AI initiation, whereas states with a higher number of plans with cost-sharing above the mean had lower rates of AI initiation. States with higher mean costsharing (in USD) were also associated with higher probabilities of AI initiation. Finally, a greater spread in deductible or monthly drug premium costs was associated with lower adjusted probabilities of $\mathrm{AI}$ as initial therapy among women in those states.

\section{Discussion}

Substantial residual geographic variation was observed in the likelihood of using an AI as initial endocrine therapy among this nationally representative cohort of 16,541 older breast cancer survivors, despite controlling for patient SES, breast cancer treatment, and other potential confounders. The adjusted probability of choosing an AI versus tamoxifen as initial adjuvant endocrine therapy ranged from $57.3 \%$ in Wyoming to $92.6 \%$ in the District of Columbia. Nearly one-third of such (adjusted) statelevel variation was explained by differences in characteristics of Medicare Part D plans available in the subjects' state of residence.

Our results indicate that a substantial portion $(30 \%)$ of state-to-state variation in the probability

\begin{tabular}{|c|c|}
\hline Variable & $\begin{array}{l}\text { Coefficient } \\
(P \text { Value })\end{array}$ \\
\hline \multicolumn{2}{|l|}{ Age, y } \\
\hline $65-74$ & Ref \\
\hline $75-84$ & $0.030(.207)$ \\
\hline $85-89$ & $-0.058(.144)$ \\
\hline \multicolumn{2}{|l|}{ Race } \\
\hline Non-African American, non-Hispanic & Ref \\
\hline African American & $-0.042(.398)$ \\
\hline Hispanic & $-0.052(.400)$ \\
\hline \multicolumn{2}{|l|}{ Comorbidity level } \\
\hline 0 & Ref \\
\hline 1 & $0.028(.294)$ \\
\hline$\geq 2$ & $0.040(.200)$ \\
\hline \multicolumn{2}{|l|}{ Low-income subsidy beneficiary } \\
\hline No & Ref \\
\hline Yes & $0.119(.000)$ \\
\hline \multicolumn{2}{|c|}{ Residence in high per capita income zip code } \\
\hline No & Ref \\
\hline Yes & $0.091(.002)$ \\
\hline \multicolumn{2}{|l|}{ Residence in rural county } \\
\hline No & Ref \\
\hline Yes & $-0.035(.307)$ \\
\hline \multicolumn{2}{|l|}{ Surgery type } \\
\hline Breast-conserving surgery & Ref \\
\hline Mastectomy & $0.183(.000)$ \\
\hline \multicolumn{2}{|l|}{ Surgery year } \\
\hline 2006 & Ref \\
\hline 2007 & $-0.027(.228)$ \\
\hline \multicolumn{2}{|l|}{ Chemotherapy } \\
\hline No & Ref \\
\hline Yes & $0.507(.000)$ \\
\hline $\begin{array}{l}\text { Density of radiation oncologists in } \\
\text { county }\end{array}$ & $0.238(.041)$ \\
\hline Density of elderly women in county & $-0.179(.010)$ \\
\hline Wald likelihood ratio test ${ }^{\mathrm{b}}$ & $574(.000)$ \\
\hline
\end{tabular}

aThe estimation also adjusted for the patient's state of residence (coefficients not shown; see Figure 1 for state-specific adjusted probabilities of having an aromatase inhibitor [AI] as the initial endocrine therapy drug of choice). Positive (negative) coefficients indicate factors that increase (decrease) the likelihood that a patient with that trait would start endocrine therapy with an Al as opposed to tamoxifen. Standard errors have been adjusted to account for clustering effects (ie, multiple observations on patients for a given surgeon).

bThe Wald likelihood ratio test, a statistic commonly used for testing the joint significance of the explanatory variables, was used to compare and inform model selection. 


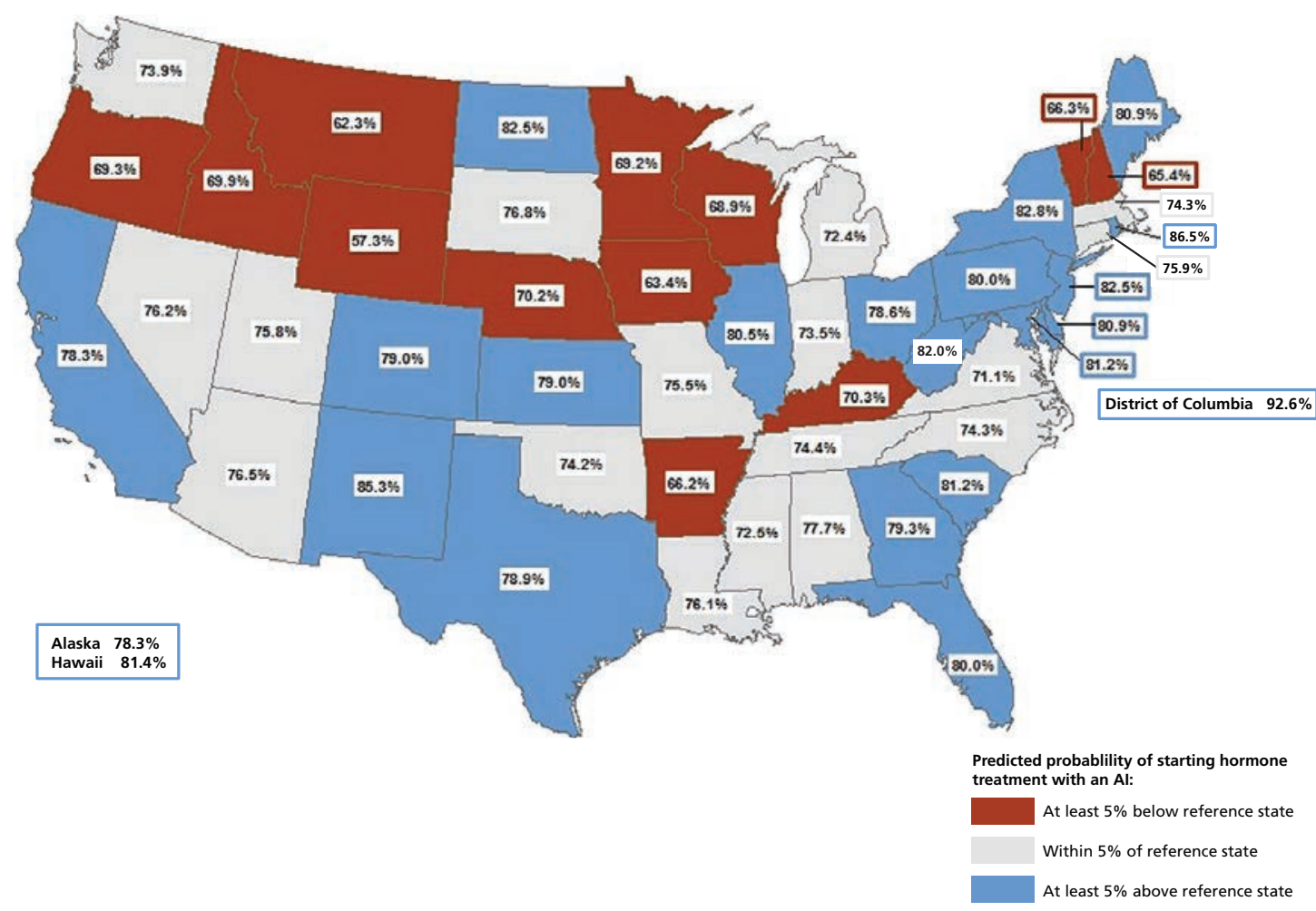

Figure 1. Predicted probability a of initiating therapy on an aromatase inhibitor (Al; anastrozole, exemestane, letrozole) by state of residence. ${ }^{a}$ Adjusted for age, race, comorbidity, chemotherapy, year and type of breast surgery, low-income subsidy status, zip code per capita income, rural county of residence, population of women aged $\geq 65$ years in county of residence, total radiation oncologists per capita in county of residence for surgery year, and clustering within surgeon (ie, multiple patient observations for each surgeon).

of initiating therapy with an AI was explained by the characteristics of Part D plans offered in each state. These findings are consistent with those reported by Zhang et $\mathrm{al}^{2}{ }^{2}$ wherein differences in design of Part D plan benefits were shown to partially explain variation in the use of more expensive, often brand name, medications. Of the 16 Part D plan characteristics initially considered in our second-stage model, 5 significantly contributed to explaining geographic variation in use of AI as initial endocrine therapy. Each of these variables captured different dimensions of cost-sharing, deductibles, and premiums that are plan-specific rather than drug-specific.

Medication cost is an important factor in patient utilization of and adherence to medications in general, ${ }^{14,15}$ and adherence to breast cancer adjuvant endocrine therapy in particular. ${ }^{16}$ Women using AIs are more likely to report experiencing financial hardship related to their treatment compared with those taking tamoxifen. ${ }^{12}$ Women with prescription drug coverage and those with higher incomes are more likely to be prescribed AIs. ${ }^{17}$ Among women with
$\mathrm{HR}+$ breast cancer and Medicare Part D coverage, out-of-pocket costs were higher for AIs, and this was associated with higher rates of nonadherence. ${ }^{18-20}$ In addition, nonadherence increased among AI users without an LIS when they reached the Part D coverage gap..$^{20}$ The prospect of reaching the "donut hole" combined with dramatic increases in the cost of Medicare Part D premiums since 2009 for the most commonly used plans ${ }^{5}$ raises fears that more breast cancer survivors will find themselves with less than full coverage for AI agents after the plan's enactment compared with before. This is particularly concerning given evidence that 1 in 5 patients with breast cancer and insurance use up all or most of their savings during the course of treatment. ${ }^{21}$

Surprisingly, variation in AI-specific monthly drug cost was not among the final list of Part D plan characteristics that were significantly associated with state variation in $\mathrm{AI}$ use. The question remains as to what other factors might account for the approximately $70 \%$ of unexplained variance in initial endocrine therapy that is associated with state of res- 
State Variation in Breast Cancer Therapy

\section{Table 3. Effect of Characteristics of Part D Plans on State Variation}

\begin{tabular}{|lc}
\hline State-Specific Part D Plans Characteristic & Coefficient (SE) \\
\hline Number of drug plans with monthly premium above the mean & $0.075(0.020)$ \\
Mean drug cost-sharing (top quartile value, in dollars) & $0.091(0.030)$ \\
Spread between the top and bottom quartiles of deductible & .003 \\
Number of drug plans with cost-sharing above the mean & $-0.021(0.007)$ \\
Spread between the top and bottom quartiles of the monthly drug premium & $-0.069(0.026)$ \\
Adjusted $R^{2}$ & $-0.023(0.012)$ \\
\hline
\end{tabular}

The dependent variable in this second-stage estimation is the state fixed effects obtained from the first-stage individual-level estimation ( $\mathrm{n}=50$ ). These fixed effects capture the independent influence of state of residence on the probability of choosing an aromatase inhibitor as initial endocrine therapy, after controlling for potential patient-level confounders and clustering effects. Results are based on forward stepwise regression, applied to a larger pool of state-level variables, which included all variables capturing variation in benefit plan design (see text for detailed description), as well as number of Part D Prescription Drug Plans (PDPs) in each state and PDP enrollment in each state. None of these additional variables were selected for inclusion for the best-fitting stepwise likelihood maximization process.

idence. It is likely that a portion of the residual unexplained variation in medication utilization is due to practice style of prescribing providers who are correlated with geographic location. ${ }^{22,23}$ For example, AI use in our cohort is correlated with a more aggressive approach to treatment, as evidenced by the positive associations between initial AI choice, chemotherapy, and mastectomies. There is no reason to believe that factors such as tumor size, stage, HER2 status, and HR status, which drive these choices in treatment, would vary so widely from state to state. In addition, even after controlling for urban setting and population of elderly women, we observed a higher utilization of AI therapy in areas with a greater density of cancer specialists (radiation oncologists). It is also possible that direct and indirect drug marketing efforts impact the variability we observed.

ASCO has recommended since 2005 that adjuvant treatment for postmenopausal women with $\mathrm{HR}+$ breast cancer should include an $\mathrm{AI} .{ }^{4}$ At the time our cohort of patients was starting treatment, there were large high-quality studies ${ }^{24,25}$ that showed longer disease-free survival and fewer distant recurrences in women treated for 5 years with AIs compared with tamoxifen. Other studies published around the same time demonstrated that switching to an $\mathrm{AI}$ after 2 to 3 years of tamoxifen was superior to taking tamoxifen alone for 5 years, but studies documenting that upfront AI for 5 years and sequential tamoxifen and AI had similar outcomes were not published until after 2010. Therefore, in 2006 and 2007, physicians were left to "guess" which strategy was better for patients, perhaps using their assessments of the importance of the additional benefit of AIs, such as reduction in several commonly recognized adverse effects associ- ated with tamoxifen, including thromboembolism and endometrial carcinoma. ${ }^{26}$ Data from our study indicate that $76.6 \%$ of patients started on an $\mathrm{AI}$ as initial endocrine therapy, suggesting rapid adoption of this class of medications as preferred initial therapy. The NCI now states in its Health Professional guidelines that AIs have become the first-line adjuvant therapy for postmenopausal women. ${ }^{27}$ Consistent with these statements, ASCO recommends use of tamoxifen alone only in instances where women have a contraindication to or are intolerant of AIs. ${ }^{4}$ There is no reason to think that contraindications to or intolerances of AIs would vary substantially at the state level.

Although evidence suggests that both the utilization of and adherence to prescription medications overall have increased since the implementation of the Medicare Part D program in 2006, ${ }^{28-30}$ the proportion of women prescribed AIs as initial treatment in this Part D era was remarkably similar to that reported for patients treated before the advent of the program. ${ }^{13,17,31}$ Our study finds that differences in treatment by age and SES persisted after implementation of Part D, with older patients and those with multiple comorbidities being less likely to initiate therapy with AIs. Consistent with previous studies documenting significant urban/rural differences in breast cancer treatment, women living in rural areas were also less likely to use AIs as their initial therapy. ${ }^{6,13,17}$

Our study has a number of limitations that merit comment. Our analysis focuses on choice of an AI as the initial therapy. There are, however, 2 strategies for AI use in women in with early-stage estrogen receptor-positive breast cancer: upfront AI treatment or sequencing to an $\mathrm{AI}$ after 2 to 3 years of 
tamoxifen treatment. Given that our analysis focuses on the former approach, we are unable to make any statements about the "appropriateness" of treatment. There are also legitimate clinical reasons not to prescribe the more expensive but more effective oral AI as initial therapy, such as the increased risk of osteoporosis associated with these agents. Unfortunately, we were unable to assess osteoporosis or differences in accessibility to its treatments in our study. It is unlikely, however, that concerns about the incidence of this or other AI-related side effects would explain the systematic and substantial variability in prescription patterns by state of residence observed in our sample. In addition, given that this study is part of a larger project focusing on the early years of Medicare Part D implementation, our analysis examines the experience of patients with breast cancer during 2006 to 2008, a time that precedes the availability of generic alternatives to AIs (2010-2011). Finally, we purposely opted against a more direct, individual-level analysis of the relationship between AI initiation and characteristics of Part D plans chosen by women in our sample. Given that nationwide administrative data sets, such as Medicare, do not contain information that would have enabled us to account and correct for participation (in Part D) and self-selection (in specific Part D plans) biases, any results from such a more direct approach would have been spurious. By relying on a mixed, individual-ecologic, 2-stage approach, we provide unbiased estimates of the effect of (differences in characteristics of) Part D plans available in each state on state-level variation in AI use, net of individual socioeconomic, demographic, and disease confounders.

\section{Conclusions}

This study raises concerns that, although Medicare Part D has improved older patients' access to prescription drugs, it did relatively little to reduce geographic variation in treatment patterns. Variations in the characteristics of Part D plans available in different states accounted for an important share of the remaining geographic variability, a finding of increasing relevance in an era when there is a rapidly expanding number of oral anticancer therapies. Although the cost of AIs has declined substantially since the recent advent of generic alternatives, our findings provide an important framework for understanding the effect of Part D plan variability on treatment of other malignancies using expensive oral chemotherapy agents. A more recent example might be CML, which has numerous costly oral drugs approved for use, and until one of them (imatinib) recently came off patent, none of the drugs had affordable generic versions. Insurance plans should be structured to encourage utilization of the agents with best evidence of efficacy and to facilitate access to these drugs for the patients who need them.

Our findings also reveal significant variations in healthcare provision not explained by individual sociodemographic and disease characteristics, or differences in state-level access to care or Part D plan characteristics. Studies that strive to understand the causes of this unexplained variation will be important as we work to maximize value in the US healthcare systems.

\section{References}

1. Donohue JM, Morden NE, Gellad WF, et al. Sources of regional variation in Medicare Part D drug spending. N Engl J Med 2012;366:530-538.

2. Zhang Y, Baicker K, Newhouse JP. Geographic variation in Medicare drug spending. N Eng J Med 2010;363:405-409.

3. Early Breast Cancer Trialists' Collaborative Group (EBCTCG). Effects of chemotherapy and hormonal therapy for early breast cancer on recurrence and 15-year survival: an overview of the randomized trials. Lancet 2005;365:1687-1717.

4. Winer EP, Hudis C, Burnstein HJ, et al. American Society of Clinical Oncology technology assessment on the use of aromatase inhibitors as adjuvant therapy for postmenopausal women with hormone receptorpositive breast cancer: status report 2004. J Clin Oncol 2005;23:619-629.

5. Nattinger AB, Pezzin LE, McGinley EL, et al. Patient costs of breast cancer endocrine therapy agents under Medicare Part D vs with generic formulations. Springerplus 2015;4:54.

6. Nattinger AB, Laud PW, Bajorunaite R, et al. An algorithm for the use of Medicare claims data to identify women with incident breast cancer. Health Serv Res 2004;39(6 Pt 1):1733-1749.

7. Eicheldinger C, Bonito A. More accurate racial and ethnic codes for Medicare administrative data. Health Care Financ Rev 2008;29:27-42.

8. Klabunde CN, Legler JM, Warren JL, et al. A Refined comorbidity measurement algorithm for claims-based studies of breast, prostate, colorectal, and lung cancer patients. Ann Epidemiol 2007;17:584-590.

9. United States Census Bureau. Census 2000, Summary File 3. Available at: https://www.census.gov/census2000/sumfile3.html. Accessed on June $10,2017$.

10. U.S. Department of Health and Human Services, Health Resources \& Services Administration, Bureau of Health Workforce. Area Health Resources Files. Available at: https://datawarehouse.hrsa.gov/topics/ahrf aspx. Accessed October 31, 2017.

11. United States Census Bureau. Metropolitan and Micropolitan. Available at: https://www.census.gov/programs-surveys/metro-micro.html. Accessed May 26, 2010.

12. Medicare Plan Finder. Medicare.gov Web site. Available at https://www. medicare.gov/find-a-plan/questions/home.aspx. Accessed October 31 , 2017. 
13. Pezzin LE, O'Neil MB, Nattinger AB. The economic consequences of breast cancer adjuvant hormonal treatments. J Gen Intern Med 2009;24(Suppl 2):446-450.

14. Streeter SB, Schwartzberg L, Husain N, Johnsrud M. Patient and plan characteristics affecting abandonment of oral oncolytic prescriptions. J Oncol Pract 2011;7(3 Suppl):46s-51s.

15. Goldman DP, Joyce GF, Zheng Y. Prescription drug cost sharing: associations with medication and medical utilization and spending and health. J Am Med Assoc 2007;298:61-69.

16. Hershman DL, Tsui J, Meyer J, et al. The change from brand-name to generic aromatase inhibitors and hormone therapy adherence for earlystage breast cancer. J Natl Cancer Inst 2014;106:pii: dju319.

17. Yen TW, Czypinski LK, Sparapani RA, et al. Socioeconomic factors associated with adjuvant hormone therapy use in older breast cancer survivors. Cancer 2011;117:398-405.

18. Neugut AI, Subar M, Wilde ET, et al. Association between prescription co-payment amount and compliance with adjuvant hormonal therapy in women with early-stage breast cancer. J Clin Oncol 2011;29:2534-2542.

19. Sedjo RL, Devine S. Predictors of non-adherence to aromatase inhibitors among commercially insured women with breast cancer. Breast Cancer Res Treat 2011;125:191-200

20. Riley GF, Warren JL, Harlan LC, Blackwell SA. Endocrine therapy use among elderly hormone receptor-positive breast cancer patients enrolled in Medicare Part D. Medicare Medicaid Res Rev 2011;1:E1.

21. Bosanquet N, Sikora K. The economics of cancer care in the UK. Lancet 2004;5:568-574.

22. Fisher ES, Bynum JP, Skinner JS. Slowing the growth of health care costslessons from regional variation. N Engl J Med 2009;360:849-852.
23. Sirovich B, Gallagher PM, Wennberg DE, Fisher ES. Discretionary decision making by primary care physicians and the cost of U.S. health care. Health Aff (Millwood) 2008;27:813-823.

24. Howell A, Cuzick J, Baum M, et al. Results of the ATAC (Arimidex, Tamoxifen, Alone or in Combination) trial after completion of 5 years' adjuvant treatment for breast cancer. Lancet 2005;365:60-62.

25. The Breast International Group (BIG) 1-98 Collaborative Group, Thürlimann B, Keshaviah A, et al. A comparison of letrozole and tamoxifen in postmenopausal women with early breast cancer. N Engl J Med 2005;353:2747-2757

26. Amir E, Seruga B, Niraula $S$, et al. Toxicity of adjuvant endocrine therapy in postmenopausal breast cancer patients: a systematic review and metaanalysis. J Natl Cancer Inst 2011;103:1299-1309.

27. National Cancer Institute. Breast Cancer Treatment (PDQ)-Health Professional Version. Available at: https://www.cancer.gov/types/breast/hp/ breast-treatment-pdq\#section/all. Accessed October 31, 2017.

28. Kennedy JJ, Maciejewski M, Liu D, Blodgett E. Cost-related nonadherence in the Medicare program: the impact of Part D. Med Care 2011;49:522 526.

29. Schneeweiss S, Patrick AR, Pedan A, et al. The effect of Medicare Part D coverage on drug use and cost sharing among seniors without prior drug benefits. Health Aff (Millwood) 2009;28:w305-316.

30. Donohue JM, Zhang Y, Lave JR, et al. The Medicare drug benefit (Part D) and treatment of heart failure in older adults. Am Heart J 2010;160:159_ 165.

31. Wu XC, Lund MJ, Kimmick GG, et al. Influence of race, insurance, socioeconomic status, and hospital type on receipt of guideline-concordant adjuvant systemic therapy for locoregional breast cancers. J Clin Oncol 2012;30:142-150.

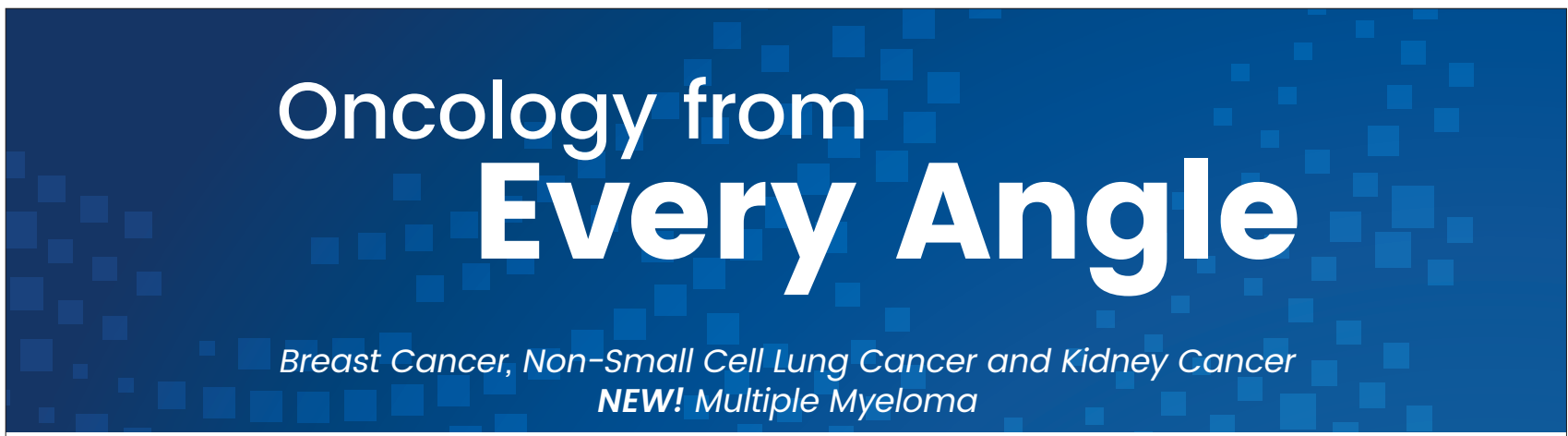

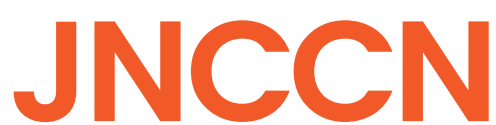

A NEW ONLINE INTERACTIVE RESOURCE

Discover more at JNCCN360.org
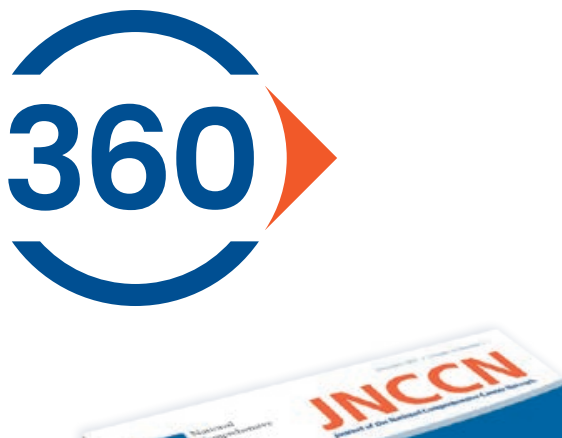

From the publishers of JNCCN 